\title{
TRASTORNOS DE PERSONALIDAD EN CONSUMIDORES DE DROGAS: DATOS PRELIMINARES
}

\author{
PERSONALITY DISORDERS IN LICIT AND ILLICIT \\ DRUGS CONSUMERS: PRELIMINARY STUDY
}

\author{
Da Silva, F.; Pérez García, C.; Costa Ball, D.; Scafarelli, L. y Del Arca, D. \\ Universidad Católica del Uruguay, Uruguay
}

\begin{abstract}
Resumen: Se estudia la Comorbilidad entre consumo de drogas legales e ilegales y el tipo de Trastorno de Personalidad en una muestra de 320 adultos: a través de Entrevista Clínica Psiquiátrica (estandarizada y normatizada) a pacientes que concurrieron a un servicio especializado en adicciones. Hay un predominio del sexo masculino, solteros, con edad de inicio en el consumo de drogas de 20 años y de consulta -promedio- de 35 años. El $50 \%$ de las consultas fue por alcohol, en ambos sexos. Las drogas ilegales más frecuentes fueron marihuana y cocaína. Se encontraron diferencias de género, entre ellas, el consumo de sedantes propio de las mujeres y el de marihuana en los hombres, así como un perfil de personalidad diferente.En los Trastornos de Personalidad predominaron los del grupo B. El consumo de drogas ilegales se asocia a los Trastornos Disocial y Límite. En los hombres se agrega el Narcisista y en las mujeres el Histriónico y Dependiente.
\end{abstract}

Palabras clave: Drogas Legales e Ilegales; Adicción. Perfil de Consulta; Trastornos de Personalidad.

\begin{abstract}
Comorbility between licit and illicit drugs and personality disorders is studied in a sample of 320 adults who attended specialized addictions service. There is a predominance of single-male participants with a starting age at 20 on drug consumption while consultation begins around 35. Fifty percent of the consultations correspond to alcohol in both sexes. The more frequent Illicit drugs were marijuana and cocaine. Gender differences were found. Firstly, the use of sedatives in women and of marijuana in men, and, secondly different personality profiles. The more predominant personality disorders were the ones of the cluster $\mathrm{B}$. The consumption of illicit drugs is associated with the borderline and antisocial personality disorders. In men, this was found concurrently with narcissistic personality disorder and in women, with the dependent and histrionic personality disorders
\end{abstract}

Key words: Licit and Illicit Drugs. Addiction. Consultation Profile. Personality Disorders

\section{INTRODUCCIÓN}

Esta comunicación es la primera de un estudio que se está realizando en la ciudad de Montevideo (Uruguay), sobre comorbilidad entre consumo de sustancias y Trastorno de Personalidad. Se estudia una población que asiste a un centro de tratamiento privado a través de entrevistas semiestructuradas psicológicas y psiquiátricas.

Caracterizar el consumo de drogas legales e ilegales en los pacientes con Trastornos de
Personalidad permitiría medir el impacto que producen las mismas en los pacientes y poder identificar perfiles de consumo en función de su edad, sexo, estado civil, nivel de educación, edad de inicio, tipo de droga de consumo, respuesta al tratamiento, etc. Este conocimiento posibilitaría detectar los grupos más vulnerables frente al consumo y generar políticas de promoción y prevención, más allá del interés en la psicopatología.

A pesar de que contamos con datos del país y de la región, aún quedan vacíos desde

Correspondencia: Prof. Dr. Fredy da Silva, Facultad de Psicología de la Universidad Católica del Uruguay. Correo Electrónico: fdasilva@ucu.edu.uy 
el punto de vista epidemiológico acerca de la incidencia del uso de alcohol y drogas ilegales en relación a los Trastornos de Personalidad.

Este primer informe de investigación tiene como objetivo describir los aspectos relevantes de los dos tópicos centrales de esta investigación: abuso de consumo de sustancias y trastornos de personalidad.

\section{ASPECTOS REFERIDOS}

A LOS TRASTORNOS DE PERSONALIDAD Y LAS DROGAS

La relación entre Trastornos por Abuso de Sustancias y Trastornos de Personalidad es compleja. Los diagnósticos de Trastornos de Personalidad parecen aumentar la vulnerabilidad para otras patologías, entre ellas el abuso y la adicción a sustancias. Las investigaciones epidemiológicas han encontrado una alta relación entre ambos (Kessler R.C., McGonagle, K., Zhao, S., Nelson, CB, Hughes, M., Eshleman, S., Wittchen, H., Kendler, K., 1994), y se puede hablar de comorbilidad o patología dual (Cervera, 1999).

En un estudio realizado en 1986 se analizaron 212 pacientes, la frecuencia de pacientes con Trastornos de Personalidad era mayor entre los pacientes con Trastornos por consumo de sustancias (Gándara, J. J. \& Medina, M. A., 1998).

Regier, D., Farmer, M., Rae, D., Locke, B., Keith, S., Judd, L. y Goodwin, F. (1990) estudiaron la comorbilidad de trastornos mentales y el consumo de alcohol y otras drogas de abuso en una muestra de 20.291 adultos mayores de edad. Encontraron que el $37 \%$ de los alcoholistas presentaban un trastorno comórbido.

Skodol, A., Oldham, J. y Gallaher, P. (1999) estudiaron la comorbilidad del eje II en consumidores de sustancias en pacientes referidos al tratamiento de trastorno de personalidad. Encontraron que el $60 \%$ de los pacientes con trastorno de personalidad presentaban también trastorno por consumo de sustancias.

Otro estudio realizado en pacientes con doble diagnóstico internados en una unidad especializada encontró en una muestra de 100 pacientes una prevalencia de Trastornos de Personalidad de 53\% con predominio del trastorno border, $74 \%$ y trastorno disocial, $66 \%$ (Roos, S, 2003)

Havassy, B., Alvidrez, J. y Owen, K. (2004) estudiaron los Trastornos Psiquiátricos y Trastornos por consumo de sustancias en una muestra de 226 pacientes comórbidos. Encontrando una alta prevalencia de enfermedades mentales graves en los pacientes con trastorno por consumo de sustancias.

Grant, B., Stinson, F., Dawson, D., Chou, P., Ruan, J. y Pickering, R. (2004), estudiaron la concurrencia del Trastorno por consumo de alcohol y drogas en pacientes con Trastorno de Personalidad en población de Estados Unidos., con una muestra de 43.000 pacientes mayores de 18 años. Encontraron que el 28,6\% de los alcoholistas y un $47,7 \%$ entre los consumidores de sustancias ilegales tenían por lo menos un trastorno de personalidad. Los más frecuentes fueron el antisocial, histriónico y dependiente.

Fernández-Montalvo, J. y Lorea, L. (2007) llevan a cabo una recensión sobre la relación de la comorbilidad de los trastornos de personalidad con el consumo de cocaína hasta el año 2007. La prevalencia de los Trastornos de Personalidad alcanza aproximadamente al $66 \%$, presentando una mayor prevalencia los Trastornos de Personalidad del grupo B asociados a los Trastornos antisocial y límite.

En todos los casos se observa un peor pronóstico cuando existe un diagnóstico de personalidad comórbido y mayores dificultades en el tratamiento. Si bien existen trabajos que muestran la relación entre ciertos trastornos de personalidad y uso de sustancias tanto en la cantidad y número de tóxicos consumidos, estos son escasos en general.

Estudios prospectivos evidencian que la respuesta al tratamiento es peor en los pacientes con doble diagnostico que en aquéllos con único diagnóstico y que los costos económicos son mayores ya que aumenta el número de abandonos del tratamiento a la vez que reingresan en las clínicas con mayor frecuencia y consultan en urgencia reiteradamente.

La comorbilidad psiquiátrica exige un correcto diagnóstico y abordaje de ambas patologías, así como un seguimiento ambulatorio prolongado de los pacientes para su adecuada rehabilitación.

\section{ESTADO DE LA CUESTIÓN RESPECTO A LAS ADICCIONES EN URUGUAY}

El uso indebido de drogas legales e ilegales, se ha convertido en una situación que afecta a una muy amplia porción de la población mun- 
dial.

En América Latina existe una marcada tasa de prevalencia, constituyendo uno de los fenómenos mas graves de los últimos tiempos, ya que afecta a niños y jóvenes de todos los sectores y de todas las clases sociales. Los miembros de la Red Latinoamericana de Investigadores en Drogas (REDLA) presentaron un análisis sobre la problemática del consumo de drogas, resaltando que el consumo aumenta en todos los países y a la vez disminuye la percepción de riesgo (CICAD, 2008). Se trata de un problema que excede el ámbito de la salud, que involucra factores sociales, culturales, económicos y políticos.

Según el Informe Mundial 2007 de la Oficina de Naciones Unidas contra las Drogas y el Delito la prevalencia en el consumo de Marihuana es del 3,8\% en personas de 15 a 64 años. En el caso de la Cocaína es del 0,3\% (ONUDD, 2007).

El Observatorio Interamericano de Drogas de la Oficina de Naciones Unidas Contra las Drogas y la Comisión Interamericana para el Control del Abuso de Drogas, realizó durante los años 2006 - 2007 el Primer Estudio Comparativo Sobre Consumo de Drogas y Factores Asociados en Población de 15 a 64 años en Argentina, Chile, Bolivia, Ecuador, Perú y Uruguay. Los objetivos eran determinar la prevalencia de consumo de droga, estimar el porcentaje de población con signos de dependencia, entre otros. (CICAD, 2008).

Los principales resultados del informe destacan que la prevalencia del consumo de Alcohol en Ecuador representa la tasa más baja $(56,8 \%)$ y en Chile la más alta $(76,8 \%)$ de los seis países. Uruguay alcanza el $67,2 \%$, uno de cada diez consumidores de alcohol del último mes presenta consumo problemático. En cuanto a la edad de inicio $25 \%$ comienza a los 15 años o antes, $50 \%$ a los 16 años o menos y $75 \%$ a los 18 o menos (CICAD, 2008).

La Marihuana es la droga más consumida a nivel mundial y entre la población joven, llegando al $80 \%$ del total de consumidores de drogas ilícitas. Según el Informe Anual 2007 del la Oficina de Naciones Unidas contras las Drogas y el Delito (ONUDD), la prevalencia mundial del consumo es del 3,8\% en personas de 15 a 64 años. Este promedio es superado en los seis países del reporte del Primer Estudio Comparativo Sobre Consumo de Drogas y Factores Asociados en Población de 15 a 64 años donde se informa de una prevalencia del 4,8\%.(CICAD, 2008).

Estudiando los datos para Uruguay, se observa que la prevalencia aumenta al $6 \%$ en la población general y al $8.9 \%$ si se trata solo de hombres. Es más notoria en la población de entre 15 y 34 años $(10,1 \%)$ frente a un $1,8 \%$ en los mayores de 34 años. En cuanto a la edad de inicio el $25 \%$ comienza con 16 años o menos, el $50 \%$ con 18 años o menos y el $75 \%$ con 20 años o menos. (CICAD, 2008).

La Cocaína, según el Informe Anual 2007 del la Oficina de Naciones Unidas contras las Drogas y el Delito (ONUDD), tiene una prevalencia de consumo del 0,3\% en personas de 15 a 64 años. Este promedio también es superado en los seis países del reporte el Primer Estudio Comparativo Sobre Consumo de Drogas y Factores Asociados en Población de 15 a 64 años (ONUDD, 2007) donde se informa de una prevalencia del $1,4 \%$.

En Uruguay, se observa una prevalencia del $1,68 \%$, siendo del $2,75 \%$ en hombres y $0,78 \%$ en mujeres. Nuevamente la población entre 15 y 34 años es la de mayor prevalencia $(2,89 \%)$ frente a un $0,46 \%$ en mayores de 34 años. En cuanto a la edad de inicio en Uruguay el $25 \%$ comienza con 17 años o menos, el $50 \%$ con 19 años o menos y el $75 \%$ con 21 años o menos. (CICAD, 2008).

La Pasta Base en Uruguay se caracteriza por tener un consumo en hombres del 0,64\% frente al $0,13 \%$ en mujeres. Entre los 15 y 34 años es del $0,73 \%$, siendo la edad de inicio: el $25 \%$ a los 16 o antes, el $50 \%$ con 18 o menos, el $75 \%$ con 21 o menos (CICAD, 2008).

En nuestro país el Observatorio Uruguayo de Drogas (OUD) realizó sobre 7000 encuestados la Cuarta Encuesta Nacional en Hogares sobre Consumo de Drogas entre los meses de noviembre y diciembre de 2006 en población de 12 a 65 años que residen en ciudades de más de 10.000 habitantes de todo el país. Se concluye que de cada 100 uruguayos, 13 consumieron alguna vez en la vida alguna sustancia ilegal. El perfil característico de consumo es el de hombres con edades entre 19 y 34 años que residen en Montevideo. Las mujeres uruguayas, duplican a los hombres en el consumo de Antidepresivos y Tranquilizantes (OUD, 2006). Las drogas ilegales (Marihuana, Cocaína y Pasta Base) evidencian un aumento de prevalencia en su consumo que es consistente con los resultados del Informe Mundial del 
2007 (ONUDD, 2007).

En los últimos años ha aumentado la investigación sobre la presencia de Trastornos de Personalidad en adictos a drogas. El interés por este tema radica en su importancia epidemiológica, su papel etiológico y su influencia en el pronóstico y tratamiento de la drogodependencia. El diagnóstico de ambas patologías (adicciones y trastornos de la personalidad) se ha ido incrementando (OUD, 2006). Diversas investigaciones indican que las personas con abuso de sustancias presentan una alta tasa de prevalencia de distintos trastornos de personalidad (Seivewright \& Daly, 1997). Son pocos, sin embargo, los estudios que evalúan los trastornos de personalidad en función del tóxico consumido (Skodol, Oldham \& Gallear, 1999). Por otra parte, varios estudios muestran que un diagnóstico de trastorno de personalidad en pacientes con abuso de sustancias se asocia con síntomas de mayor adicción, mayor riesgo de recaída y peores resultados en los tratamientos tradicionales (Nace \& Davis, 1993).

En Uruguay, nuestro equipo ha realizado un estudio entre 1997 y 1999 evaluando y comparando el consumo de drogas legales e ilegales en pacientes ambulatorios de una policlínica para el tratamiento del uso indebido de drogas y alcohol de la Asociación Española (TUIDAL) y el Servicio de Fármacode-pendencia del Hospital Maciel (MSP) (da Silva, F., Severino, G., Pérez, M. y Pereira, T., 2003).

En el 2006, en el VI Congreso Latinoamericano de Psiquiatría Biológica obtiene el primer premio el poster "Avances de la Investigación: Trastornos de la Personalidad y Adicciones" (Pérez, M., Costa, C. D., da Silva, F).

El presente artículo tiene como objetivo estudiar la comorbilidad (doble diagnóstico) entre el consumo de drogas legales e ilegales y el tipo de Trastorno de Personalidad, en una muestra de adultos que concurrieron voluntariamente entre los años 2000 y 2008 a un servicio ambulatorio especializado en el tratamiento del uso indebido de drogas y alcohol.

\section{METODOLOGÍA}

\section{SUJETOS}

El universo de estudio de esta muestra es la población que consulta en un servicio ambulatorio especializado en el tratamiento del uso indebido de drogas y alcohol.

De una base de datos de 2000 historias clínicas se eliminaron aquellas que no presentaban trastornos de personalidad, las consultas familiares y las de tabaquismo. Se evaluaron 320 sujetos mayores de edad que cumplieron con la condición de presentar el doble diagnóstico entre trastorno por consumo de drogas y trastorno de personalidad. La muestra esta conformada por 221 hombres y 99 mujeres de Montevideo entre 18 y 78 años de edad.

\section{MATERIALES}

Se elabora una historia clínica anónima en formato electrónico que se identifica por un número de usuario de manera de asegurar la más absoluta confidencialidad.

Se codifican las historias siguiendo el capítulo de Trastornos Mentales y del Comportamiento de la $10^{\circ}$ edición del Manual de Clasificación Internacional de Enfermedades (CIE10).

\section{PROCEDIMIENTO}

Se trata de pacientes que consultan en un servicio especializado en la asistencia y tratamiento del uso indebido de drogas y alcohol en la ciudad de Montevideo. Las entrevistas clínicas son realizadas en primera instancia por un psicólogo que recibe al paciente en su primera consulta y al menos dos psiquíatras que recaban los datos en una historia clínica normatizada (semi-estructurada) modelo. Los pacientes son entrevistados en varias oportunidades ya que continúan su asistencia en el mismo servicio. El diagnóstico surge en una reunión de equipo y en base a las entrevistas clínicas.

\section{ANÁLISIS ESTADíSTICO}

Estudio descriptivo de corte transversal donde se analizan las variables evaluadas en la muestra: frecuencias y porcentajes en las variables cualitativas y cuantitativas (sexo, estado civil, ocupación, droga de consumo y trastorno de personalidad) y medidas de tendencia central en las variables cuantitativas (edad de inicio y edad de consulta).

Para la comparación de los porcentajes de cada patrón de consumo de droga, trastorno de personalidad y porcentajes de tipo de trastornos de personalidad por tipo de consumo de droga, se utilizó el programa estadístico SPSS 14 para Windows. 


\section{RESULTADOS}

En primer lugar estudiamos las variables sociodemográficas en la muestra de 320 sujetos estudiados. En segundo lugar analizamos la frecuencia de consumo de droga y por último la frecuencia de los trastornos de personalidad y su relación con el tipo de abuso de sustancias.

El 30,9\% de la muestra (99 sujetos) son mujeres y el 69,1\% (221 sujetos) hombres. La tabla 1 que muestra el perfil sociodemográfico, evidencia que el $48,8 \%$ de los sujetos son solteros, $31 \%$ casados o vive en unión libre, $18 \%$ divorciados o separados y $2,4 \%$ viudos. En relación a su actividad, el 39,5 \% no trabaja (34, $2 \%$ hombres y $51,5 \%$ mujeres).

En suma, los sujetos con trastorno por consumo de sustancias y de personalidad se caracterizan mayoritariamente por ser hombres en una relación de 3 a 1 , solteros, y con un porcentaje mayor de ocupación laboral (65,8\% contra un $48 \%$ de las mujeres).

La edad de consulta se observa en la tabla 2, la edad promedio es de 35,2 años. Un $25 \%$ consultan a los 24 años o menos, el 50\% a los 31 años o menos y el $75 \%$ a los 43 años o menos. Los hombres consultan a edades más tempranas que las mujeres en promedio a los 33,8 años y las mujeres a los 38,2 años. El $75 \%$ de los hombres ha consultado a los 41 años o menos, en cambio el $75 \%$ de las mujeres lo hacen recién a los 49 años.

En la tabla 3 se muestra la edad de inicio en el consumo de drogas. La edad promedio para la muestra de ambos sexo es de 20,6 años. La media para hombres es de 18,8 años frente a los 24,9 para mujeres. Se observa que un $25 \%$ de los consumidores de droga (legal o ilegal) inician el consumo a los 15 años o menos, el $50 \%$ a los 18 años o menos y el $75 \%$ a los 24 años o menos. Es importante destacar que entre los 11 y los 24 años el $75 \%$ de los sujetos ya se han iniciado en el consumo. Las diferencias por sexo muestran que el $75 \%$ se inició entre los 11 y 20 años para los hombres y entre los 11 y 31 para las mujeres.Un $25 \%$ de los consumidores hombres inician el consumo a los 15 años o menos y las mujeres demoran un año más. El $50 \%$ de los hombres lo hace por primera vez a los 17 años o menos, mientras que las mujeres a los 21 años. El 75\% de los hombres comienza el consumo a los 20 años o menos y las mujeres a los 31 .

En la tabla 4 se puede apreciar el "peso" de drogas legales e ilegales en la muestra. El 50,3 $\%$ de personas con Trastorno de Personalidad

\begin{tabular}{|c|c|c|}
\hline \multicolumn{3}{|c|}{ Table 1} \\
\hline \multicolumn{3}{|c|}{ Distribucidn por atida cill } \\
\hline \multicolumn{3}{|c|}{ Erthong } \\
\hline & H & Y \\
\hline Bham & 14 & dH. \\
\hline Grodownón ibo & 9 & 319 \\
\hline Yos & 7 & 22 \\
\hline 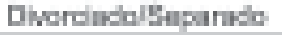 & $\Delta$ & 170 \\
\hline Tathi & Hin & 101 \\
\hline
\end{tabular}

\begin{tabular}{|c|c|c|c|}
\hline \multicolumn{4}{|c|}{ 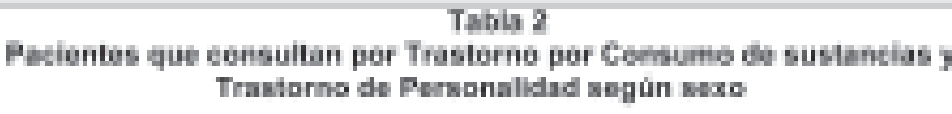 } \\
\hline \multicolumn{4}{|c|}{ 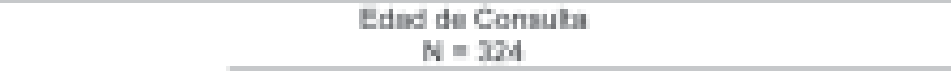 } \\
\hline & Muintru & $\begin{array}{c}\text { Hanbur } \\
\text { nH }\end{array}$ & $\begin{array}{l}\text { Mupuriti } \\
\text { ag }\end{array}$ \\
\hline Movit & $35 y$ & 38 & HA, \\
\hline$P_{n}$ & 24 & 14 & 26 \\
\hline 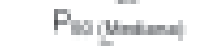 & 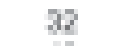 & $M 1$ & 36 \\
\hline$P$ & 43 & 41 & 41 \\
\hline Ranres & 担- & 1L - TE & $18-\pi$ \\
\hline
\end{tabular}


que consultan son consumidores de alcohol, el resto corresponde a la sumatoria del consumo de todas las drogas ilegales. Analizando el consumo de alcohol por sexo encontramos que más de la mitad de las personas que consultan y tienen un Trastorno de Personalidad son mujeres.

La tabla 5 presenta el estudio de frecuencia del tipo droga de consumo e identifica las sustancias de mayor consumo: 50,3\% alcohol, $17,2 \%$ cocaína y derivados, $15,9 \%$ marihuana, $4,1 \%$ sedantes y $10,6 \%$ policonsumo. El resto de las drogas presentan un porcentaje de uso inferior al $2 \%$.

Analizando la droga de consumo en función del sexo (tabla 5), se encuentra que el alcohol tiene un porcentaje de consumo en mujeres del $53,5 \%$ frente al $48,9 \%$ de los hombres. En cuanto a la marihuana y a la cocaína y sus derivados los hombres superan a las mujeres en una relación de aproximadamente 2 a 1 Las mujeres presentan un porcentaje importante de consumo de sedantes que los hombres prácticamente no registran $(12,1 \%$ frente al $0,5 \%)$. El policonsumo tiene porcentajes muy similares en ambos sexos (10\%).

En la figura 1 se presentan los datos correspondientes a todos los sujetos de la muestra con la distribución de las frecuencias absolutas en cada Trastorno de Personalidad y para cada droga de consumo. El alcohol tiene la frecuencia absoluta más alta. El $70 \%$ de los

\begin{tabular}{|c|c|c|c|}
\hline \multicolumn{4}{|c|}{$\begin{array}{c}\text { Tatla } 1 \\
\text { Edad da linklo por scow }\end{array}$} \\
\hline \multicolumn{4}{|c|}{$\begin{array}{l}\text { Edad th nod } \\
\qquad \mathrm{H}=\mathrm{240}\end{array}$} \\
\hline & Muasta & $\begin{array}{l}\text { Hortres } \\
\text { ist }\end{array}$ & $\begin{array}{l}\text { Myeres } \\
71\end{array}$ \\
\hline Hetct & 201 & 166 & 24 \\
\hline $\mathrm{P}_{\mathrm{s}}$ & 15 & 10 & 16 \\
\hline Ponthisy & 15 & 17 & 21 \\
\hline$P_{5}$ & 24 & 30 & 31 \\
\hline Fango & $11-6 j$ & $11=\mathrm{ta}$ & \\
\hline
\end{tabular}

\begin{tabular}{|c|c|c|c|c|c|c|}
\hline \multicolumn{7}{|c|}{ 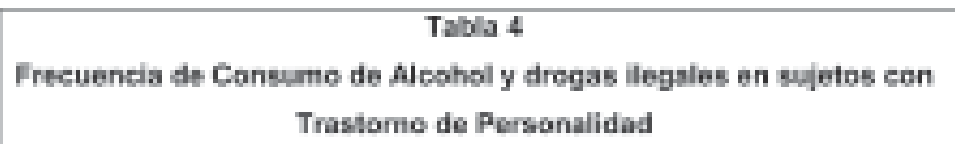 } \\
\hline & \multicolumn{2}{|c|}{ 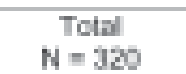 } & \multicolumn{2}{|c|}{$\begin{array}{l}\text { Hombes } \\
N=2 i\end{array}$} & \multicolumn{2}{|c|}{$\begin{array}{l}\text { Mujoras } \\
\text { Nest }\end{array}$} \\
\hline & $\mathbf{n}$ & 5 & $\Pi$ & $\$$ & $\Pi$ & 5 \\
\hline Dopa logal íaboholi & 167 & 50 & 100 & 489 & 52 & 535 \\
\hline Doges ibgy & 154 & 47 & 117 & $51 \div$ & 40 & 45 \\
\hline Tokil & sol & 100 & 221 & 1001 & 99 & 100 \\
\hline
\end{tabular}

\begin{tabular}{|c|c|c|c|c|c|c|}
\hline \multicolumn{7}{|c|}{ 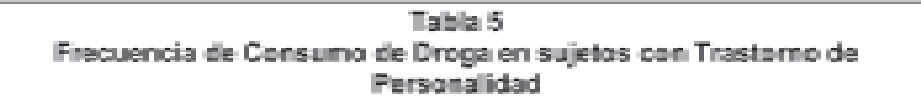 } \\
\hline & \multicolumn{2}{|c|}{$\begin{array}{c}\text { Total } \\
\mathrm{N}=\mathrm{se}\end{array}$} & \multicolumn{2}{|c|}{$\begin{array}{c}\text { Horbues } \\
\text { M }=201\end{array}$} & \multicolumn{2}{|c|}{$\begin{array}{l}\text { Wuenes } \\
N=99\end{array}$} \\
\hline & m & 㛃 & 1 & 要 & n & 2 \\
\hline Alouhal & 1E1 & 503 & 119 & 489 & 5 & 555 \\
\hline Maihuena & 51 & 159 & 43 & 19.5 & $\mathrm{E}$ & a. 1 \\
\hline Cocaina y terwados & 56 & 172 & 42 & 1 19ja & 13 & 131 \\
\hline Setantese Hondtoos & 13 & 41 & 1 & 15 & 12 & 121 \\
\hline Folounsum: & 34 & +06 & 24 & 109 & 10 & 10,1 \\
\hline guss & E & $1 \equiv$ & 3 & 14 & \pm & 31 \\
\hline Tol 3 & 320 & 100 & 221 & 120 & 99 & 100 \\
\hline
\end{tabular}


consumidores de alcohol se distribuye en 5 tipos de Trastornos de Personalidad, en orden decreciente: dependiente $(17 \%)$, mixto $(16 \%)$, histriónico (16\%), inespecífico (12\%) y narcisista ( $9 \%)$. A su vez el $68 \%$ de los consumidores de marihuana se distribuye en 4 tipos de Trastornos de Personalidad: Inespecífico (22\%), disocial $(20 \%)$, límite $(14 \%)$ y mixto $(12 \%)$. En cuanto al consumo de cocaína y sus derivados el $68 \%$ de los consumidores se distribuye preferentemente en tres trastornos de personalidad: límite (26\%), disocial (24\%) y narcisista (18\%). Por último el $80 \%$ del policonsumo se distribuye entre límite (32\%), disocial (24\%) y narcisista $(24 \%)$.
En la tabla 6 se presenta la frecuencia de ocurrencia de Trastornos de Personalidad en la muestra. Los trastornos con mayor frecuencia de aparición son en orden decreciente (ver figura 2): 15\% trastorno límite (F.60.3), 13,4\% trastorno disocial (F60.2), 13,1\% trastorno mixto (F61), 12,5\% trastorno narcisista (F60.8), 11,9\% trastorno histriónico (F60.4), 11,6\% trastorno inespecífico (F60.9) y 11,3\% trastorno dependiente (F60.7)

La tabla 7 muestra la frecuencia de cada Trastorno de Personalidad en función del sexo (Ver también figura 3). En los hombres el 73\% de los Trastornos de Personalidad se agrupan en cinco trastornos: $16 \%$ presenta trastorno

Fipure 4

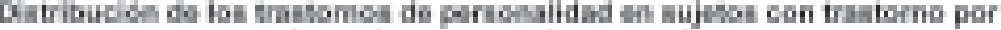

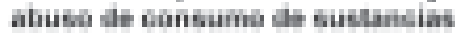

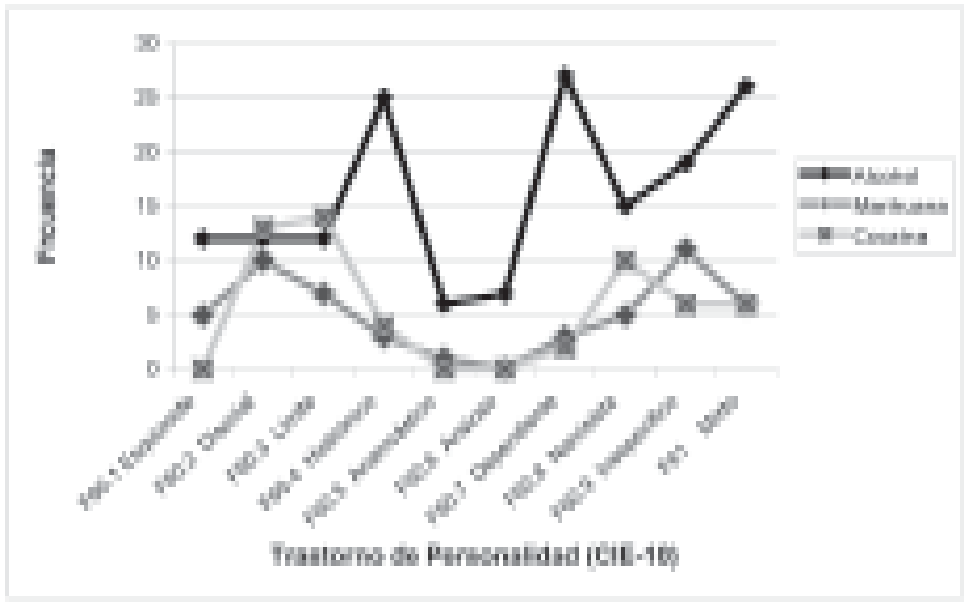

\section{Twhin 4}

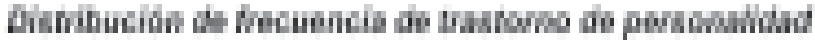

\begin{tabular}{|c|c|c|}
\hline 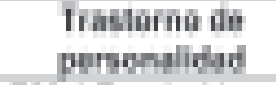 & A & $\begin{array}{c}\text { Pondhing } \\
\text { 4 }\end{array}$ \\
\hline 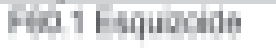 & $n$ & his \\
\hline FWI 2 Dhodi & 41 & 114 \\
\hline FHA Limin & Ith & $4 n$ \\
\hline Phy Hhithin & $M$ & 114 \\
\hline 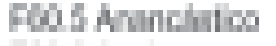 & b & il \\
\hline PhI Ginth & 8 & H. \\
\hline 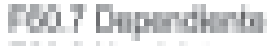 & $y$ & 119 \\
\hline Fha Him HWil & 19 & AA \\
\hline 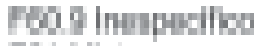 & $M$ & 119 \\
\hline Fil Mishi & 14 & +11 \\
\hline Tanl & ni & 111 \\
\hline
\end{tabular}


mixto (F61), 15\% trastorno límite (F60.3), 15\% trastorno narcisista (F60.8), 14\% trastorno disocial (F60.2) y el $13 \%$ trastorno inespecífico (F60.9). Las mujeres presentan el $28 \%$ trastorno histriónico (F60.4), 18\% trastorno dependiente (F60.7), 12\% trastorno disocial (F60.2) y 14\% trastorno límite (F60.3).

Con el objetivo de analizar cómo se relaciona la edad de consulta con el tipo de droga que el sujeto consume, dividimos la distribución de la edad de consulta en seis franjas lo más equitativamente posible. En la tabla 8 se muestra la frecuencia y proporción de sujetos en cada franja de edad que construimos.

En la Figura 4 se aprecia la cantidad de pacientes con Trastorno de Personalidad y la droga de consumo por franja de edad. La edad de consulta oscila en la muestra entre 18 y 78 años (tabla 2), dividimos este rango de edad en seis categorías y en cada una de estas

Figura 5

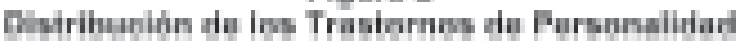

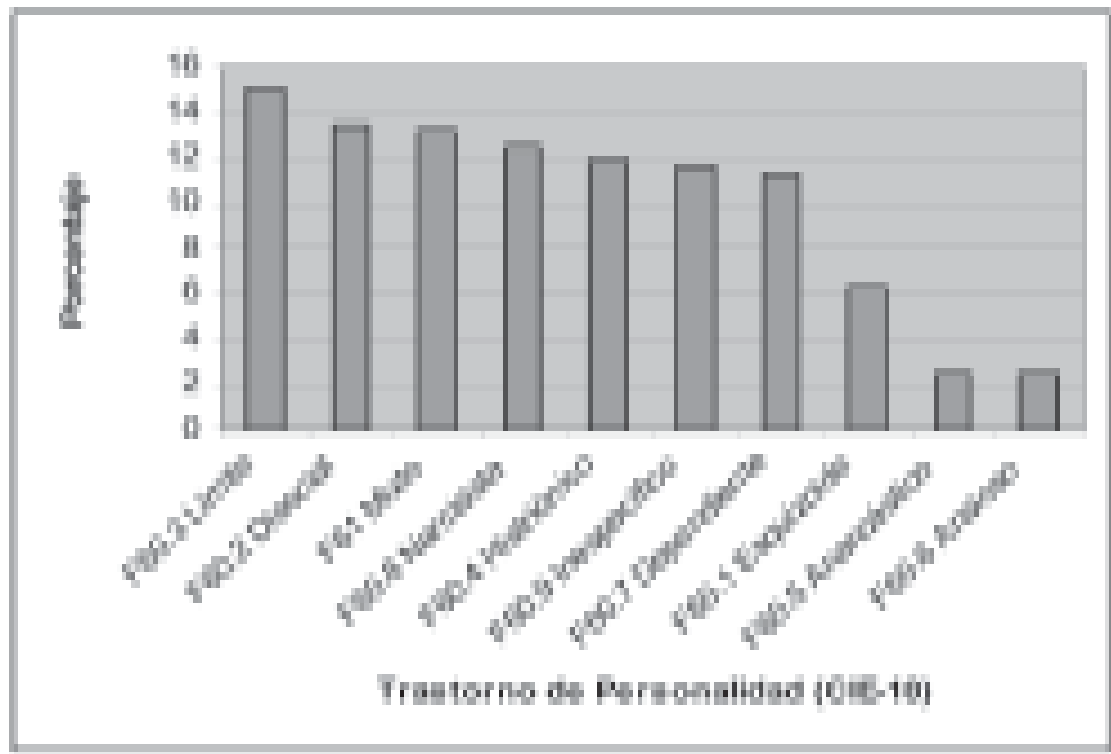

\begin{tabular}{|c|c|c|c|c|}
\hline \multicolumn{5}{|c|}{ 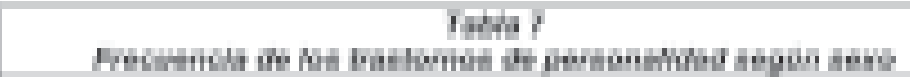 } \\
\hline \multirow[t]{2}{*}{ 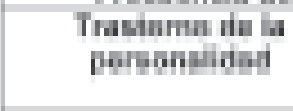 } & \multicolumn{2}{|c|}{ Homirus } & \multicolumn{2}{|c|}{ Anjurits } \\
\hline & $\mathbf{H}$ & $\mathrm{H}$ & H & th \\
\hline H I Iнमаны & th & $\mathrm{Fy}$ & i & at \\
\hline 4n & 19 & 140 & $1 \mathrm{H}$ & H. \\
\hline 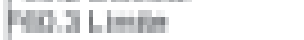 & II & 141 & II & ill \\
\hline What Hakndm & 10 & $d n$ & IH & my \\
\hline has nurphon & F & 21 & it & 4 \\
\hline 변 Arthis & . & 17 & 1 & In \\
\hline 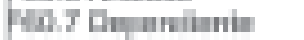 & Ii & m & III & 18 \\
\hline mo pimphin & iㅓ & $14+$ & H & 14 \\
\hline 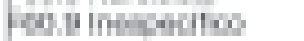 & n & $A T$ & H & 14 \\
\hline Hil Mrin & Hit & in $\mathrm{x}$ & 1 & H I \\
\hline
\end{tabular}


Fipura 3

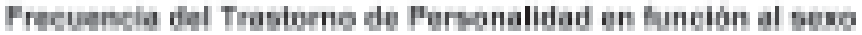

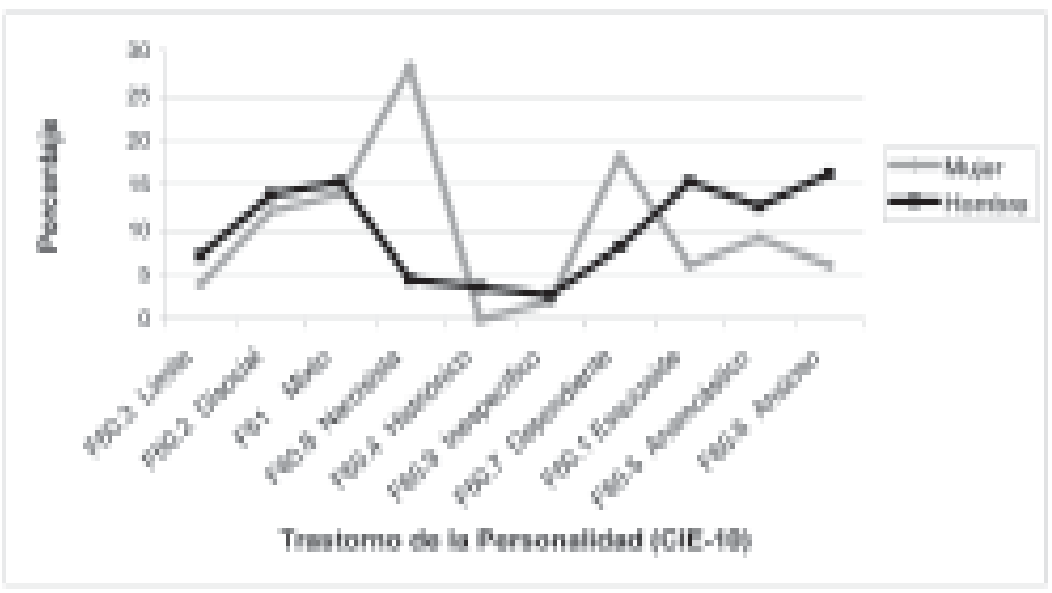

Tathlin it

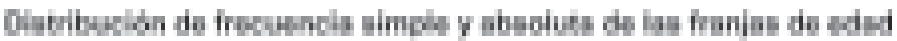

\begin{tabular}{|c|c|c|c|c|}
\hline 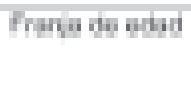 & ท & H & 4 & $\begin{array}{c}\text { ⿱ㅗ } \\
\text { mumbink }\end{array}$ \\
\hline $15-n$ & H & m & MA & 17. \\
\hline มาม & III & 111 & IIII & 배교 \\
\hline$M-31$ & 44 & Whin & thi & bat \\
\hline 柴的骨 & III & 111 & IfII & 패이 \\
\hline 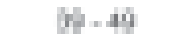 & $M$ & wi & 17i & bis \\
\hline If & เม & ำด & HIII & in \\
\hline Whit & wo & & Wi & \\
\hline
\end{tabular}

obtuvimos una frecuencia absoluta aproximadamente del $18 \%$ de los consultantes. En el alcohol más del $20 \%$ de los que consultan tienen entre 18 y 22 años pero se destaca como a partir de la franja de 26 a 31 años el porcentaje de consulta llega al $45 \%$ y de ahí en más se mantiene un aumento sostenido. En el caso del consumo de marihuana encontramos una consulta del $35 \%$ entre los 18 y 22 años, que decrece paulatinamente con el incremento de la edad. Para la cocaína el $25 \%$ pertenece a la franja etárea de 18 a 22 años, llegando a un pico de consulta entre los 23 y 25 años (30\%) para decrecer a partir de los 26 años. En cuanto a los sedantes casi no hay consulta entre los 18 y los 30 años (menor al 5\%) pero esta se incrementa de manera progresiva a partir de los 32 a 39 años (10\%) para mantenerse en ese porcentaje. Dadas las diferencias por sexo, que hacen que las mujeres tengan un protagonismo muy específico estos datos serán analizados en la figura 5 . El policonsumo tiene un $15 \%$ entre los 18 y 22 años, llega al $20 \%$ entre los 20 y 25 años para luego decrecer en forma paulatina con el aumento en edad.

En las figuras $\mathbf{5}$ y $\mathbf{6}$ desglosaremos el porcentaje de consumo por franja de edad y droga según sexo. En la figura 5 se aprecia el perfil de consumo de mujeres destacándose el consumo de alcohol y sedantes. Entre los 23 y 25 años duplican a los hombres en el porcentaje de consulta por alcohol (30\% frente a un $15 \%$ del sexo masculino). Entre los 26 y 31 llegan al $50 \%$ de la consulta por alcohol. Después de los 32 años se nivela la consulta en ambos sexos. En cuanto a los sedantes las mujeres consultan desde los 18 a los 26 años con un porcentaje inferior al $10 \%$, pero a partir de los 32 años aumenta de manera importante superando el $20 \%$ de consulta. Prácticamente no se 

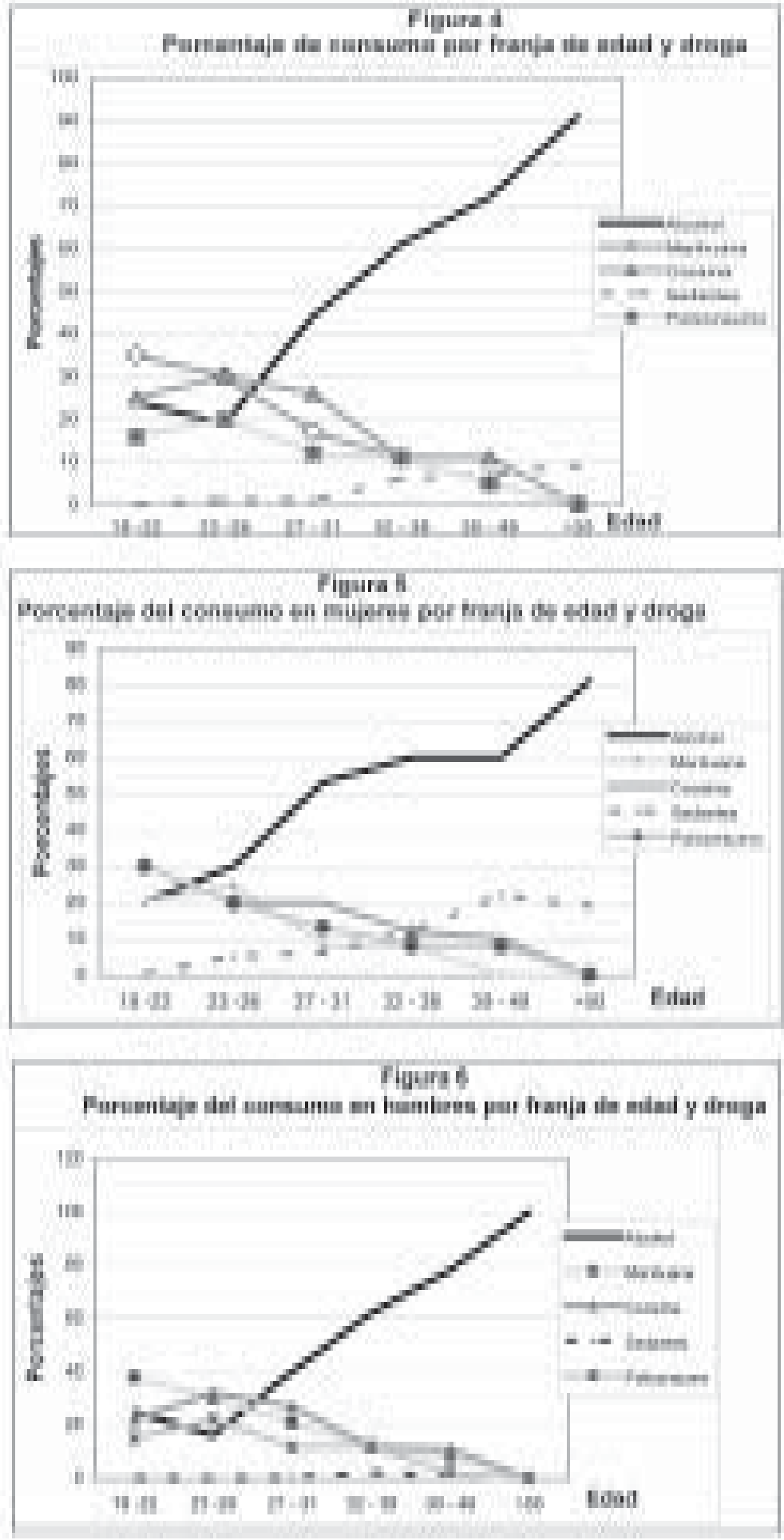

registra consulta por consumo de esta droga en hombres (figura 6). Comparando el consumo de marihuana en hombres y mujeres aproximadamente el $40 \%$ de los hombres consultan entre los 18 y 22 años frente al $20 \%$ en las mujeres de la misma edad. Las mujeres presentan un notorio descenso en el porcentaje de consumo de marihuana después de los 26 años. La cocaína 
en las mujeres sigue un patrón muy similar al de la marihuana, en cambio en el hombre entre los 23 y 25 años presenta un $32 \%$ de consulta para luego decaer paulatinamente.

\section{DISCUSIÓN}

Del estudio sociodemográfico rescatamos que la consulta tuvo un claro predominio del sexo masculino ya que de cada 3 hombres que consultaron lo hizo solo una mujer. En cuanto al estado civil predominaron los solteros siendo prácticamente la mitad de la muestra. Si sumamos divorciados y viudos este porcentaje es de prácticamente el $70 \%$. Solo el $31 \%$ está casado o en unión libre. La edad de inicio en el consumo de drogas tuvo un promedio en los 20 años, siendo un poco anterior en los hombres (19 años frente a 25 de las mujeres). En relación a la edad de consulta tuvo un promedio de 35 años. El $75 \%$ de la muestra consultó antes de los 42 años en el caso de los hombres y antes de los 50 años en el caso de las mujeres. Analizando estos datos podemos plantear que los hombres inician el consumo a menor edad pero también la consulta es más precoz.

Analizando los datos obtenidos sobre la droga de consumo encontramos que el $50 \%$ de las personas con Trastornos de Personalidad que consultaron por consumo de sustancias lo hicieron por alcohol, con una diferencia no significativa entre ambos sexos. En cuanto a las drogas ilegales la consulta fue por consumo de marihuana y cocaína en casi el mismo porcentaje (32\% y $35 \%$ respectivamente de las drogas ilegales). Las diferencias se establecen al tener en cuenta el sexo ya que un $20 \%$ de los hombres consultaron por consumo de marihuana frente a un $8 \%$ de mujeres. Para la cocaína la consulta fue de un $19 \%$ de hombres contra un $13 \%$ de mujeres. El policonsumo fue muy similar para ambos sexos, aproximadamente un $10 \%$. Si, se establecen claras diferencias para el consumo de sedantes que fue de $12 \%$ en mujeres y solo el $0,5 \%$ en hombres.

En suma el alcohol es la droga más importante, casi sin diferencia de género, mientras que en el hombre predomina la consulta por consumo de marihuana y en la mujer aquella por sedantes. En otras drogas no hay mayores diferencias.

Al estudiar el consumo de droga por franja de edad encontramos que la consulta por consumo de alcohol se incrementa en ambos sexos en función de la edad. A medida que aumenta la edad aumenta la probabilidad de que la consulta sea por alcohol así como disminuye la de drogas ilegales. En la mujer antes de los 32 años su porcentaje de consulta aumenta más que en los hombres, en cambio en estos el porcentaje de consulta es mayor después de los 40 años.

En referencia a los Trastornos de Personalidad hay que destacar la predominancia de trastornos del grupo o cluster B en los pacientes que consultan por consumo de sustancias. Siete de estos Trastornos de Personalidad representan aproximadamente el $90 \%$ de los sujetos con abuso de sustancias (límite, disocial, mixto, narcisista, histriónico, inespecífico y dependiente). Aproximadamente el $73 \%$ de los hombres presentan trastornos de personalidad tipo mixto, límite, narcisista, disocial e inespecífico. En las mujeres el $73 \%$ presenta trastornos histriónico, dependiente, límite y disocial.

En suma hombres y mujeres comparten el trastorno disocial y límite. Diferenciándose los hombres por presentar también los trastornos narcisista, inespecífico y mixto. Las mujeres, en cambio, el trastorno histriónico y dependiente. O sea que habría un perfil propio para cada género.

Al relacionar los Trastornos de Personalidad con el consumo de alcohol encontramos que 5 trastornos estaban presentes en el $70 \%$ de los consumidores. Estos son: dependiente, mixto, histriónico, inespecífico y narcisista. En cambio para la marihuana predominaron el inespecífico, disocial, limite y mixto. Para el caso de la cocaína los más frecuentes fueron el límite, disocial y narcisista.

Vinculando el Trastorno de Personalidad con la característica de legalidad o no de la droga de consumo encontramos que en las ilegales (marihuana, cocaína, policonsumo) aparecen con mayor frecuencia y entidad los trastornos disocial y límite (del grupo B).

Es preciso señalar como limitaciones del estudio, por un lado las propias de la muestra, ya que se la puede considerar sesgada al estar conformada por pacientes que consultan en un servicio privado de salud, lo que implica un nivel socioeconómico predeterminado (medio y medio alto). Por otro lado creemos que si bien el diagnostico clínico puede ser adecuado dada la experiencia de los técnicos que lo realizaron y el mecanismo de supervisión utilizado, sería muy importante contar con escalas o tests de personalidad en busca de una validez convergente con 
el criterio de diagnostico psiquiátrico.

A pesar de las limitaciones descritas, este trabajo aporta aspectos importantes sobre la relación de los Trastornos de Personalidad y el consumo de drogas para una población de pacientes de Montevideo.

Queda para futuros estudios continuar con el estudio de prevalencia, caracterizando el trastorno de personalidad en relación al perfil de consumo de cada droga, los períodos de abstinencia, la frecuencia de recaídas, así como hacer estudios de regresión de los datos para describir modelos con vistas a predecir evolución y pronóstico.

\section{REFERENCIAS}

Cervera G, Bolinches F, Valderrama JC. (1999). Trastornos de la personalidad y drogodepen-dencias. Trastornos Adictivos, 1 (2), 91-99.

CICAD (2008). Elementos orienta-dores para las políticas Públi-cas sobre drogas en la sub-región: primer estudio compa-rativo sobre consumo de dro-gas y factores asociados en población de 15 a 64. UN-OID

da Silva, F., Severino, G., Pérez, M. y Pereira, T (2003). Carac-terísticas del Uso de Drogas en Centros de Tratamiento de Montevideo - Uruguay. OPTAR Consultado en 20/10/2008 en http://www. iin.oea.org/foro40.pdf.

Fernández-Montalvo, J. y Lorea, L. (2007). Comorbilidad de la adicción a la cocaína con los trastornos de la personalidad. Anales del Sistema Sanitario de Navarra, 30 (2), 225-231.

Gándara, J. J. \& Medina, M. A. (1998). Comorbilidad. En M. Bernardo \& M. Roca (Eds.), Trastornos de la personalidad. Evaluación y tratamiento (pp. 97-122). Barcelona: Masson.

Grant, B, Stinson, F, Dawson, D., Chou, P., Ruan, J. y Pickering, R. (2004). Co-occurrence of 12-Month Alcohol and Drug Use Disorders and Personality Disorders in the United States. American Medical Association, 61, 361-368.

Havassy, B., Alvidrez, J. y Owen, K. (2004). Comparación de los pacientes con trastornos psiquiátricos y trastornos por consumo de sustancias comórbidos: repercusión en los servicios asistenciales y terapéuticos. American Journal of Psychiatry (Ed. Esp), 7, 237-243.

Kessler RC., McGonagle, K., Zhao, S., Nelson, CB, Hughes, M., Eshleman, S. Wittchen, H. Kendler, K. (1994). Lifetime and 12-month prevalence of DSM-III-R psychiatric disorders in the United States: results from the National Comorbidty Survey. Arch Gen Psychiatry, 51, 8-19.

Kofoed, L., Kania, J., Walsh, T., Akinson RM (1986). Outpatient treatment of patient of patients with substance abuse and coexisting psychiatric disor-ders. American Journal of Psy-chiatry 143(7):867-872
Kruedelbach, N., McCormick, R., Schulz, S., y Gruenich, R. (1993). Impulsivity, coping styles, and triggers of craving in substance abusers with borderline personality disorder. Journal of Personality Di-sorders, 7, 214-222.

Nace y Davis (1993). Treatment Outcome in Substance-Abu-sing Patients with a Personality Disorder. American Journal on Addictions, 2 (1), 26-33

ONUDD (2007). Oficina de Naciones Unidas contra las Drogas y el Delito. Informe Mundial sobre drogas 2007.

OUD (2006). Cuarta Encuesta Nacional en Hogares sobre Consumo de Hogares- No-viembre 2006

Pedrero Pérez, E.J. y Segura López, I (2003). Los trastornos de la personalidad en drogode-pendientes y su relación con la dificultad de manejo clínico. Trastornos Adictivos, 5(3), 229-240.

Pérez, C., da Silva, F. y C. D. Costa Ball (2006). Poster: Avances de la Investigación Trastornos de la Personalidad y Adic-ciones ( $1^{\circ}$ premio), en el VI Congreso Latinoamericano de Psiquiatría Biológica. Colonia de Sacramento, Uruguay

Regier, D., Farmer, M., Rae, D., Locke, B., keith, S., Judd, L.y Goodwin, F (1990). Comor-bidity of Mental Disorders With Alcohol and Other Drug Abuse Results from the Epidemiologic Catchment Area (ECA) Study. The Journal of the American Medical Association (JAMA), 264 (19), 2511-2518.

Ross, S., Dermatis, H., Levounis, P., y Galanter, M. (2003). A comparison between dually diagnosed inpatients with and without Axis II comorbidity and the relationship to treatment outcome.. The American Journal of Drug and Alcohol Abuse, 29(2), 263-279.

Seivewright, N. y Daly, C. (1997). Personality disorder and drug use: A review. Drug and Alcohol Review, 76, 235-250.

Skodol, A. E., Oldham, J. M. \& Gallaher, P. E (1999). Axis II comorbidity of substance use disorders among patients referred for treatment of personality disorders. Ameri-can Journal of Psychiatry, 156, 733-738. 